confer on it some of the various advantages which have been claimed for speciality synthetic rubbers. 'Heveaplus MG' is a graft polymer obtained by polymerizing methyl methacrylate in rubber latex, while 'Heveaplus MM' is prepared by mixing a dispersion of polymethyl methacrylate with concentrated ammonia-preserved rubber latex followed by coagulating, washing and drying. In both types, a final number indicates the percentage of (poly) methyl methacrylate present in the product. Details of the chemical and physical properties, processing, vulcanization, reinforcing power, etc., of these interesting new materials are also given.

\section{Properties of Materials at Low Temperatures}

AnNexe 1954-2 of the Bulletin of the International Institute of Refrigeration (177 Boulevard Malesherbes, Paris $17 \mathrm{e}$ ) records the proceedings of the meetings of Commissions 1 and 2 of the Institute held at Zurich in September 1953; the publication has just become available. Nineteen papers were presented and some of the topics discussed are indicated below. A paper by Long and Simon describes a helium liquefier constructed with an elastic metallic bellows in place of the conventional cylinder and piston. Van Itterbeek describes an adaptation of the Collins liquid helium cryostat to a closed cycle so that the same quantity of helium gas is circulating and the loss reduced to a minimum. An apparatus which permits of the liquefaction of hydrogen and helium in the same unit is described by Spoendlin, and Myncke describes a liquid-air plant with propane precooling circuit. Wucherer reviews the development of expansion turbines for low temperatures and quotes figures for the high efficiency attainable. Heat-transfer calculations for a crossflow regenerator are given by Hausen. Four papers deal with the low-temperature properties of metals : the mechanical proporties of ferritic steels at $-196^{\circ} \mathrm{C}$., by Allen; the effects of low temperatures on some engineering properties of metals, by Oliver; the thermal conductivities of metals below room temperatures, by Powell ; and strength of aluminium crystals at low temperatures, by Eder. Two papers deal with thermal insulation problems: one on the thermal conductivity of porous materials containing air under reduced pressure, by Paemel ; and a noto on the thermal conductivity of moist cork, by Hatton and Zaki. A survey of the field of work of Commission 2, which has for its terms of reference the study of heat transfer, insulating materials, instrumentation, and thermal properties of materials, was given by Griffiths. 'The next joint meetings of Commissions 1 and 2 will take place at Grenoble on September 24 and 25 of this year.

\section{Constants of the Stellar Velocity Ellipsoid}

WhEREAS previous derivations of the constants of the velocity ellipsoid of stars have been based on the radial velocities of the brighter stars, in a paper by D. G. Ewart (Mon. Not. Roy. Astro. Soc., 113,5 ; 1953) the derivation is based on the radial velocities of 820 stars the $H D$ photographic magnitudes of which range from $8 \cdot 5$ to $8 \cdot 6$ and the spectral types of which are $F-M$. The calculations were simplified by using large regions of the sky, which was divided into thirty-four areas, according to galactic latitude, and antipodal areas were combined to give seventeen regions over one hemisphere. The final results for the solar motion. were: $U=20 \cdot 36 \mathrm{~km}$. $/ \mathrm{sec}$.; $A=275^{\circ} 07^{\prime} ; D=$ $+40^{\circ} 44^{\prime} ; G=35^{\circ} 53^{\prime} ; g=+21^{\circ} 18^{\prime}$, with a $K$ term of $-0.22 \mathrm{~km}$. $/ \mathrm{sec}$. The mean results previously obtained by Moore and Paddock were: $U=22 \cdot 44$ km. $/$ sec. ; $A=280^{\circ} ; D=+44^{\circ} 30^{\prime}$, from which it appears that the high value of the declination of the solar apex obtained by Moore and Paddock is partly confirmed by Ewart's work. It is suggested, however, that as the stars used in earlier work on radial velocities were generally brighter than the sixth apparent magnitude-very much brighter than those used in the present investigation-there may be a magnitude variation in the solar motion.

The same division of the sky was used for the determination of the constants of the velocity ellipsoid, and a table shows the values obtained for $1 / K$, $1 / H, K / H$ and $G_{0}$ for the different zones; but it is pointed out that the only result to which any significance can be attached is that for all stars. In this case the values are $37 \cdot 24,25 \cdot 80,0 \cdot 693$ and $321^{\circ} 44^{\prime} \pm 3^{\circ} 31^{\prime}$, respectively, one main feature of which is that the ratio $K / H=0.693$ is well defined and agrees closely with proper-motion results. Delhaye's figures, published in 1952, obtained by analysing the proper motions of faint stars in the Boss General Catalogue, indicated that the phenomenon of the deviation of the vertex is confined to the nearest stars and is not exhibited by the fainter and more distant stars, and it is significant that in Ewart's investigation the deviation of the vertex is barely evident. It is suggested that there should be further examination for a possible variation of the declination of the solar apex with apparent magnitude, and also for the dependence of the deviation of the vertex on the distances of the stars used in its determination.

\section{Radioactive Tobacco Mosaic Virus}

F. L. WYND has reported on the preparation of radioactive tobacco mosaic virus (Lloydia, 16, No. 4, 233 ; 1953) by growing young tobacco plants in hydroponic culture low in phosphate for three weeks. Radioactive phosphorus in the form of orthophos. phoric acid was then added to the nutrient solutions and seven days later each plant was inoculated with the disease by rubbing the lower leaf with liquid obtained from macerated diseased leaves. Sixty-two days after inoculation, the plants were harvested and the virus recovered by differential ultracentrifugation. A purified virus suspension was obtained exhibiting a radioactivity of $2.54 \mu \mathrm{c} . / \mathrm{mgm}$. of virus phosphorus. This activity was significantly greater than those reported by previous investigators. The probable purity of the recovered virus was evidenced by electron photographs. The high radioactivity of the purified virus should permit its use in further studies of certain aspects of the physiology and biochemistry of this virus disease.

\section{The Council for the Preservation of Rural England}

THe twenty-eighth annual report of the Council for the Preservation of Rural England quotes an example from Sheffield to illustrate the need for constant vigilance in seeing that land scheduled as 'green belt' in local authorities' development plans is not used for other purposes. The Sheffield and Peak District Branch of the Council is very concerned about possible encroachments on the Sheffield 'green belt' as envisaged in the City's Development Plan. At a public inquiry in July 1953 the Branch lodged certain objections and received an assurance that various farm lands left uncoloured on the map and designated 